\title{
Differences in Kinetics of Phase Transformation of 3 Y-TZP Ceramics between Aging Test under Hydrothermal Environment and Hip Simulator Wear Test*
}

\author{
Junji IKEDA**, Mikio IWAMOTO**, Seido YARIMITSU*** \\ and Teruo MURAKAMI*** \\ **Japan Medical Materials Corporation, \\ 3-3-31 Miyahara, Yodogawa-ku, Osaka 532-0003, Japan \\ E-mail: ikedaj@jmmc.jp \\ ${ }^{* * *}$ Faculty of Engineering, Kyusyu University \\ 744 Motooka, Nishi-ku, Fukuoka-shi, Fukuoka 819-0395, Japan
}

\begin{abstract}
The phase stability of yttria ( $3 \mathrm{~mol} \%)$ stabilized tetragonal polycrystalline zirconia (3Y-TZP) has been evaluated by X-ray diffraction and laser microscope. The differences in kinetics of phase transformation in the aging test under hydrothermal environment and during the hip simulator wear test were compared. It was found that the phase transformation during aging test experiences a Mehl-Avrami-Johnson (MAJ) equation and the phase stability of tetragonal phase can be improved by controlling the sintering condition. Hip simulator wear test using zirconia femoral head with 4 different monoclinic fractions was performed against conventional UHMWPE (GUR 1050) acetabular socket up to 6 million cycles. During hip simulator testing, slight increase in monoclinic fraction of some zirconia femoral head was observed. Increasing rate of monoclinic phase depended on initial monoclinic fraction. But, from the result of measurement of UHMWPE wear rate, mutual relationship between the monoclinic fraction on zirconia femoral head and the wear rate of UHMWPE socket was not observed. From the results of laser microscope observation, in the case of phase transformation during hip simulator test, large number of small monoclinic spots were observed in contrast to the phase transformation during aging test. Moreover an increase in monoclinic fraction on the surface alone was observed significantly compared to the progress into the bulk in aging test. These facts indicate that the nucleation of monoclinic phase on the surface was enhanced by the sliding action against UHMWPE cup. Thus, it was suggested that the phase transformation during hip simulator test was affected on tribochemical reaction during sliding condition.
\end{abstract}

Key words: Zirconia, Artificial Joint, Phase Stability, Aging, Simulator Test

\section{Introduction}

Bioinert oxide ceramics such as alumina and zirconia have been used for joints and bone replacement due to their superior biocompatibility ${ }^{1,2}$. Alumina has been used for artificial joints since 1970 for its good mechanical strength and excellent corrosion resistance, and has long-term good clinical record ${ }^{3}$. Especially, alumina has extremely high scratch resistance compared to cobalt chromium molybdenum alloy that is used for typical artificial femoral heads, because of its excellent high hardness, and is service to keep initial

*Received 19 Oct., 2011 (No. 11-0631) [DOI: 10.1299/jbse.7.199]

Copyright $\mathbb{C} 2012$ by JSME 
smooth surface for long time. Besides, sliding combination of alumina and alumina for total hip replacement is attracting attention as application for more young active patients, because that combination can reduce the friction coefficient and the total amount of wear, and eliminate the Ultra high weight molecular weight polyethylene (UHMWPE) wear particles that causes osteolysis ${ }^{4}$. However, alumina is essentially brittle material. As a result, alumina has limitation of design and it is reported that catastrophic failure occurred on rare occasion in vivo use ${ }^{5}$. Current typical commercially available alumina, high purity alumina with HIP (Hot Isostatic Press) treatment according to ISO 6474, has higher fracture strength and hardness compared to the earlier generation alumina with low purity and without HIP treatment. It is reported that the fracture rate of alumina femoral head are reduced, the fracture rate of femoral head of the earlier generation alumina and that of current alumina are about $0.2 \%$ and $0.02 \%$, respectively ${ }^{6}$. So far, mechanical properties of alumina have been improved with improvement in the manufacturing process and raw material processing technology. However, concern about fracture of alumina components remains. Thus, it is necessary for more long term reliability and increase in variation of design to improve the fracture strength and toughness significantly.

On the other hand, zirconia was researched actively at 1980s for its superior fracture strength and toughness and attracted attention as alternative material to alumina ${ }^{7}$. Especially, $3 \mathrm{~mol} \%$ yttria stabilized tetragonal zirconia polycrystalline (3Y-TZP) has excellent high fracture strength over $1 \mathrm{GPa}$ in bending strength and has been used for not only artificial joints but dental parts such as fixture, abutment and crown $^{8}$. The 3Y-TZP has higher fracture strength and toughness compared to alumina due to the toughening arisen from stress induced phase transformation from tetragonal to monoclinic phase ( $\mathrm{t} \rightarrow \mathrm{m}$ transformation) $)^{9}$. Pure zirconia has three crystal structures depending on elevating temperature. Monoclinic phase, tetragonal phase and cubic phase are stable at room temperature, over 1000 degree and over 2300 degree, respectively ${ }^{10}$. It is necessary for achieving superior fracture strength to keep tetragonal phase that is stable at high temperature to room temperature. It is known that stress induced phase transformation prevents the crack propagation and enhances fracture strength and toughness, and this t-m phase transformation is accompanied with about $4 \%$ volume expansion ${ }^{11,12}$.

However, some zirconia is reported that spontaneous phase transformation occurred at comparatively low temperature under hydrothermal environment without effect on stress ${ }^{13}$. This spontaneous phase transformation is accompanied with deterioration of mechanical properties and surface smoothness, and called "Low temperature degradation (LTD)". Mechanical properties deteriorate due to the decrease of total amount of tetragonal phase for stress induced transformation and the micro-crack formation accompanied with volume expansion. Volume expansion also causes formation of swells on the surface. In 1996, the British MDA (Medical device agency) announced the warning about re-sterilization of zirconia femoral head using autoclave, because it may induce an increase in their surface roughness ${ }^{14}$. Furthermore, a high incidence of catastrophic fracture of zirconia femoral heads was reported in $2001^{15}$. This failure occurred in only zirconia femoral heads manufactured by Saint-Gobain Desmarquest. It was reported that the reason of this failure is changes in sintering method and furnace ${ }^{16}$. However, this incident is one of the reasons of discrepancy. So far, there have been both positive and negative clinical reports on zirconia femoral heads regarding the phase transformation ${ }^{17,18}$. Nowadays, the specification of the recent products is improved compared to the retrieved products reported now. The phase stability properties of zirconia femoral heads conspicuously vary depending on manufacturers and manufactured era, so several types of zirconia have been distributed and implanted.

In our previous study, it was confirmed that the phase stability of zirconia was improved by the optimization of sintering condition. However, the behavior of phase 
transformation of zirconia femoral heads in vivo and its relationship with sliding and wear have not been fully clarified yet. In this study, we carried out the aging test and the hip simulator wear test using earlier generation zirconia femoral heads against conventional UHMWPE sockets, and the kinetics of phase transformation during wear test was focused.

\section{Material and Method}

\subsection{Materials}

The earlier generation zirconia femoral heads which were not subjected to HIP treatment and the conventional (non cross-linked) UHMWPE sockets manufactured by Japan Medical Materials Corporation (JMM, Osaka, Japan) were prepared for this hip simulator study. 3Y-TZP powder with a small amount of alumina and chemical composition according to ISO 13356 was cold isostatically pressed into the cylinder with rubber mold at about $294 \mathrm{MPa}$. The pressed cylinder were cut into the shape close to final product with taper bore, and sintered in the batch furnace. After the sintering, taper bore and spherical surface were grinded into regulation shapes. And then spherical surface was polished as mirror surface below 0.02 micrometer in Ra. Average grain size and bulk density of sintered zirconia was approx. 0.3 micrometer and $6.07 \mathrm{~g} / \mathrm{cm}^{3}$, respectively. The UHMWPE sockets were shaped from GUR1050 sheet stock and sterilized by ethylene oxide gas. Diameters of the zirconia femoral heads were $22 \mathrm{~mm}$, inner and outer diameters of the UHMWPE sockets were $22.4 \mathrm{~mm}$ and $44 \mathrm{~mm}$, respectively. Images of zirconia femoral head and UHMWPE sockets are shown in Fig. 1. Three current advanced zirconia femoral heads with HIP treatment, nano crystalline zirconia, were also prepared as target of comparison in phase stability under hydrothermal environment. Average grain size and bulk density of zirconia with HIP treatment is approx. 0.2 micrometer and $6.08 \mathrm{~g} / \mathrm{cm}^{3}$, respectively.

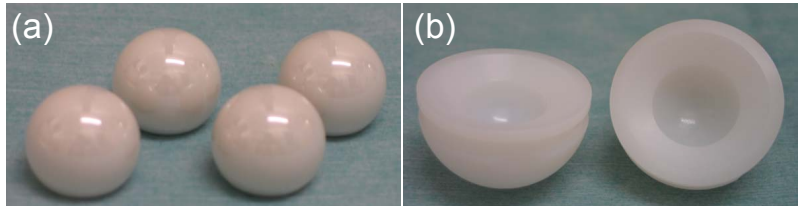

Fig. 1 Photographs of

(a) zirconia femoral heads and (b) UHMWPE sockets prepared for hip simulator test.

\subsection{Aging test}

At first, twelve zirconia femoral heads without HIP treatment were exposed in saturated vapor at $121{ }^{\circ} \mathrm{C}$ for several times in order to evaluate the phase stability under hydrothermal environment (Aging test). Aging test was performed using a tester for a pressure cooker test (ETAC PLAMOUNIT HAST CHAMBER PM422, Kusumoto Chemicals, Tokyo, Japan). Crystal structure of zirconia femoral heads before and after aging test was measured by $\mathrm{X}$-ray diffractometer (PW3050, Philips Japan, Tokyo, Japan) with $\mathrm{Cu} \mathrm{K} \alpha$ radiation $40 \mathrm{kV}$ and $35 \mathrm{~mA}$. X-ray diffraction (XRD) pattern was collected from 26 degree to 36 degree in $2 \theta$, with a step size of 0.02 degree and a counting time of 2 second per step. Typical XRD pattern of transformed zirconia are shown in Fig 2.

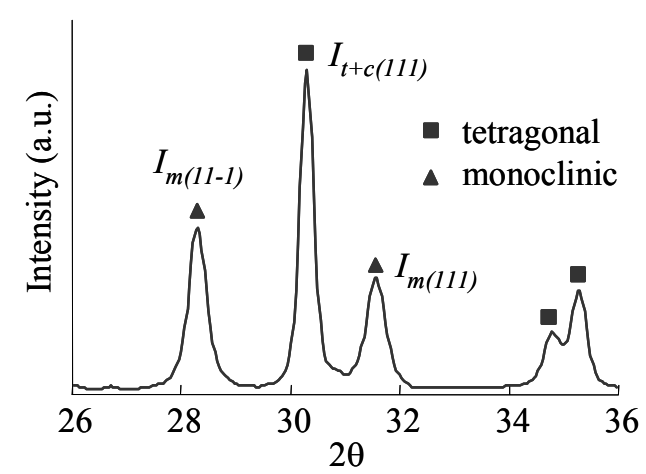

Fig. 2 Typical XRD pattern of transformed zirconia 
The monoclinic fraction was calculated according to the Garvie and Nicolson equation from the intensity ratio of tetragonal contained cubic and monoclinic phase of XRD pattern $^{20}$.

$$
X_{\mathrm{m}}(\mathrm{mol} \%)=\frac{\left(I_{\mathrm{m}(11-1)}+I_{\mathrm{m}(111)}\right)}{\left(I_{\mathrm{t}+\mathrm{c}(111)}+I_{\mathrm{m}(11-1)}+I_{\mathrm{m}(111)}\right)}
$$

Here, the monoclinic fraction $X \mathrm{~m}$ is in $\mathrm{mol} \%$, and $I_{\mathrm{j}(h k l)}$ is the intensity of the $(h k l)$ reflection band of the phase $\mathrm{j}(\mathrm{t}, \mathrm{c}$ and $\mathrm{m}$ for the tetragonal, cubic and the monoclinic phase, respectively) observed in the XRD pattern. Changes in surface morphology after aging test were observed by confocal laser microscope (OLS4100, Shimazu, Kyoto Japan),

\subsection{Hip simulator test}

Three aged and one non-aged zirconia femoral heads with four different monoclinic fractions on the surface were selected for hip simulator wear test. The monoclinic fractions of three aged zirconia femoral heads were 10, 50, and $80 \mathrm{~mol} \%$. The duration time of aging was adjusted based on the result of above aging test. The monoclinic fraction of non-aged zirconia femoral heads was $0.5 \mathrm{~mol} \%$.

The wear test was performed by the 12 channel hip simulator (AMTI Inc., Watertown, MA). Hip simulator was run according to ISO 14242 for walking pattern at $1 \mathrm{~Hz}$ up to 6 million cycles (MC). It used $27 \mathrm{wt} \%$ bovine serum with EDTA and sodium azide for lubricant of this test and the lubricant was exchanged per $0.5 \mathrm{MC}$. Temperature of lubricant was kept at $37{ }^{\circ} \mathrm{C}$ by passing through the heater/chiller unit during hip simulator test. UHMWPE sockets were soaked in lubricants at $37{ }^{\circ} \mathrm{C}$ for 4 weeks before hip simulator test. Weight loss of UHMWPE sockets were measured per $0.5 \mathrm{MC}$ and corrected by weight changes in load soak specimen. Number of each zirconia femoral heads was three and number of load soak specimens was two. Changes in the crystal structure and surface morphology of zirconia femoral heads after hip simulator wear test were evaluated per changes in lubricant (per 0.5 MC) by XRD and confocal laser microscope, respectively.

Moreover, UHMWPE wear particles were separated from collected lubricant after 0.5 $\mathrm{MC}$ and $5 \mathrm{MC}$, and configuration of UHMWPE wear particles were evaluated. Collected lubricant was combined with equivalent amount of $10 \mathrm{~N}-\mathrm{NaOH}$ water solution, and the mixed solutions were heated at $65{ }^{\circ} \mathrm{C}$ for 5 hours. And then, the solution was treated with alkali and topped with $40 \mathrm{wt} \%$ sucrose water solution and IPA(Isopropyl alcohol) water solution carefully. After centrifugal treatment of above solutions at $4000 \mathrm{rpm}$ for $5 \mathrm{~min}$, white floating substance located at the interface between sucrose water solution and IPA water solution were gathered. Sucrose water solution with $1.2 \mathrm{~g} / \mathrm{cm}^{3}$ was added, similarly, after IPA water solution was laid and centrifugal treatment was performed, white floating substances were gathered. Consecutively, methanol was added, and the supernatant solution was turn out after centrifugal treatment. Sucrose water solution with $1.05 \mathrm{~g} / \mathrm{cm}^{3}$, IPA water solutions with 0.98 and $0.90 \mathrm{~g} / \mathrm{cm}^{3}$ were laid, the white floating substance located at the interfaces among sucrose water solution and IPA water solutions were gathered after centrifugal treatment. After that, the suction filtration was performed and UHMWPE wear particles on the filter were observed using Scanning electron microscope (SEM; S-3400N). From SEM images, particle size (equivalent circle diameter) and aspect ratio were measured using commercial image analysis software.

\subsection{Refinement of crystal structure}

For characterization of crystal structure of tetragonal phase in more detail, polished 
pellets with diameter of $17 \mathrm{~mm}$ which were sintered at 9 several temperatures from 1300 and $1500{ }^{\circ} \mathrm{C}$ were prepared and their crystal structure were evaluated by X-ray diffractometer ( RINT1400V, Rigaku, Tokyo, Japan) with $\mathrm{Cu}$ K $\alpha$ radiation $40 \mathrm{kV}$ and $35 \mathrm{~mA}$. XRD pattern was collected from 26 degree to 36 degree $2 \theta$, with a step size of 0.02 degree and a counting time of $2 \mathrm{~s}$ per step. The lattice constants of tetragonal crystal and cubic ratio were determined using Rietveld anaysis. Refinements of the collected XRD spectrum were performed by the software Rietan 97-beta according to the Rietveld spectral analysis. The tetragonality of the zirconia, which is defined as the axial ratio, c/a $>1$, of the tetragonal lattice constants, could be also determined.

\section{Results}

Change in the monoclinic fraction of zirconia femoral head without HIP treatment as a function of aging time is shown in Fig. 3. It was confirmed that monoclinic fraction increased depending on aging time. Some of laser microscope images after aging test are shown in Fig. 4. Progress stages of phase transformation were divided into three stages. From zero to around $15 \mathrm{~mol} \%$, from 15 to $50 \mathrm{~mol} \%$ and over $50 \mathrm{~mol} \%$ were defined as initial stage, medium stage and late stage, respectively. In the initial stage, some small swells were observed by CLM. It is demonstrated that these observed swells were located in regions changed into monoclinic phase from the result of fine raman spectroscopic study using conforcal raman spectrometer with 1 micrometer laser probe ${ }^{21}$. The slopes of these monoclinic swells were very gradually. In the $10 \mathrm{~mol} \%$ zirconia, diameter and height of monoclinic swells were about 20 and 0.1 micrometer, respectively. The small swells spread to surroundings with increasing aging duration time up to middle stage. In the middle stage to the late stage of transformation, monoclinic fraction measured by XRD increased with increasing aging time, but laser microscope image of $80 \mathrm{~mol} \%$ zirconia femoral head are not significantly different from that of $50 \mathrm{~mol} \%$ zirconia femoral head. It was confirmed that surface was covered with monoclinic thin layer and phase transformation mainly progress into the bulk from surface with increasing aging time in the middle stage to late stage. As a reference, experimental results of nano-crystalline zirconia with HIP treatment are shown, with a gray broken line in Fig. 3.

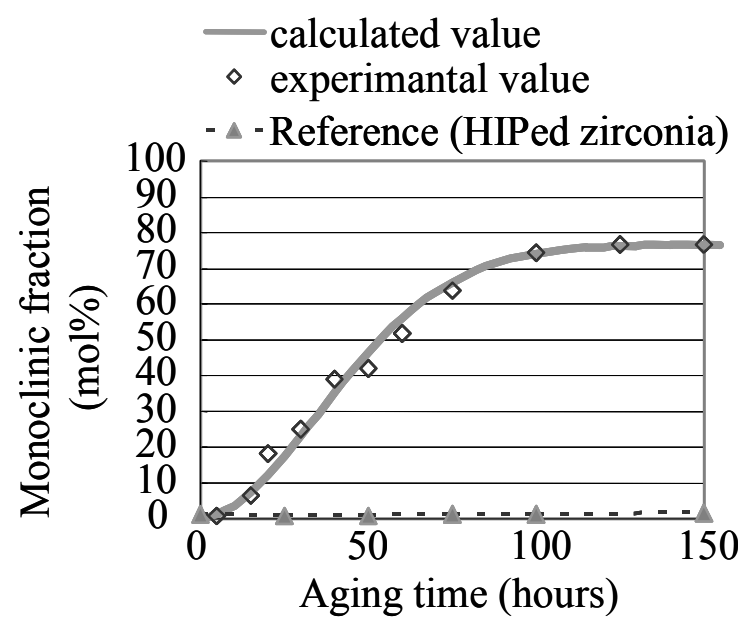

Fig. 3 Changes in the monoclinic fraction of zirconia femoral head with/without HIP treatment as a function of duration time 


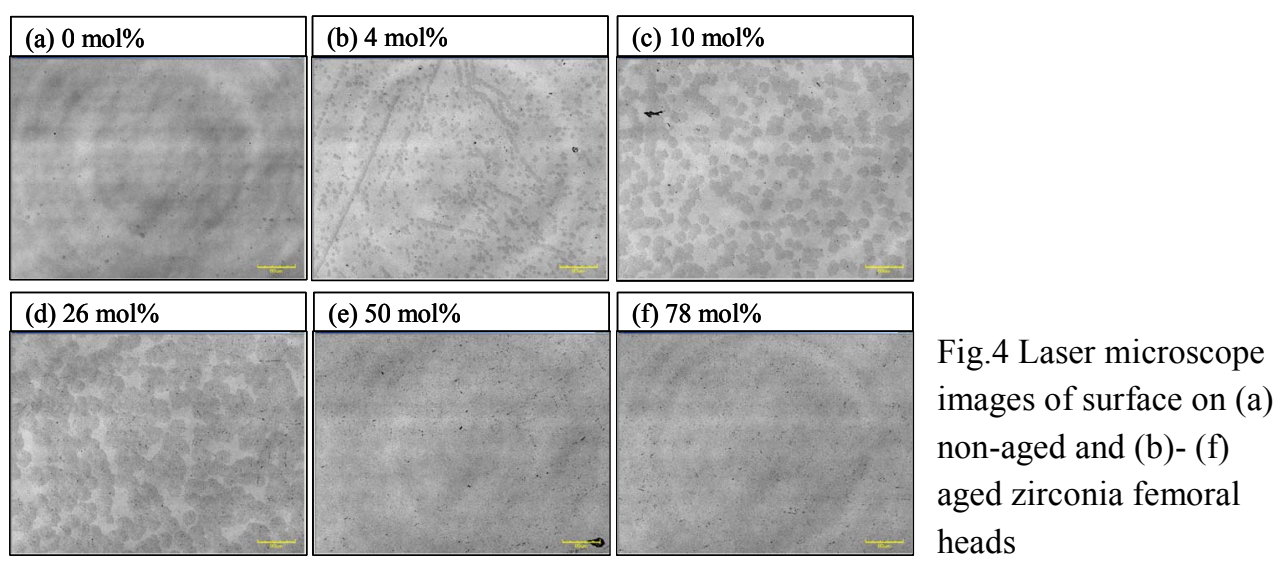

The summaries of hip simulator test are shown in Table 1. Polyethylene wear rate was 3.1 to $3.9 \mathrm{mg} / \mathrm{MC}$. No severe wear damages such as scratch and chipping on zirconia femoral heads after wear test were observed. There was no relationship between polyethylene wear rate and monoclinic fraction of zirconia femoral head. Changes in monoclinic fraction during hip simulator test are shown in Fig 5.

Table 1 Summary of Hip simulator wear test

\begin{tabular}{cccccc} 
& \multicolumn{2}{c}{$\begin{array}{c}\text { Monoclinic } \\
\text { fraction }(\mathrm{mol} \%)\end{array}$} & \multicolumn{2}{c}{ Surface roughness } & \multicolumn{2}{c}{$\begin{array}{c}\text { Polyethylene } \\
\text { Ra }(\mu \mathrm{m})\end{array}$} & Wear Rate \\
& Before & After & Before & After & $(\mathrm{mg} / \mathrm{MC})$ \\
\hline $0 \%$ & 0.2 & 13 & 0.009 & 0.012 & 3.8 \\
$10 \%$ & 10 & 18 & 0.014 & 0.012 & 3.9 \\
$50 \%$ & 50 & 52 & 0.012 & 0.013 & 3.7 \\
$80 \%$ & 78 & 77 & 0.010 & 0.012 & 3.1 \\
& & & & \multicolumn{2}{c}{ MC : million cycles }
\end{tabular}

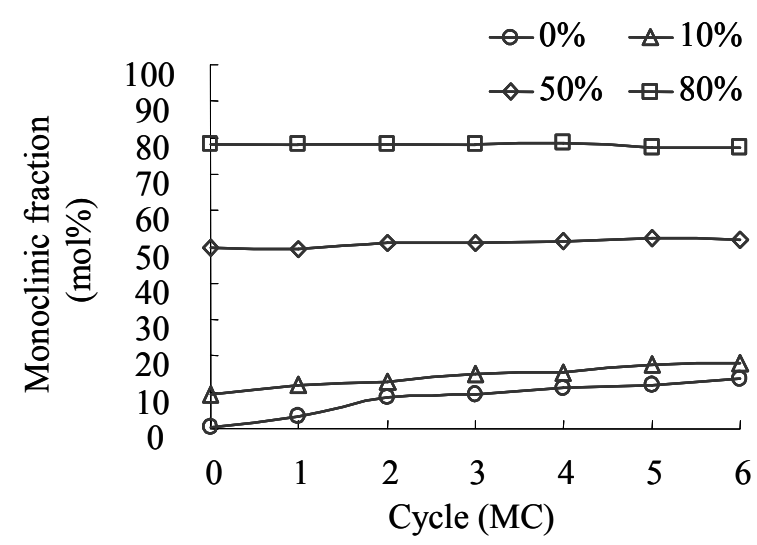

Fig. 5 Changes in monoclinic fraction during hip simulator test

In the 0 and $10 \mathrm{~mol} \%$ zirconia femoral heads, monoclinic fraction increases with increasing testing cycles, slightly. This slight phase transformation is not stress induced transformation but only a kind of spontaneous transformation, because no surface damages such as scratch by wear is observed. Laser microscope images of changing in surface morphology on the non-aged and $10 \mathrm{~mol} \%$ aged zirconia femoral heads during hip simulator test are shown in Fig 6. 
(a) Monoclinic fraction before hip simulator test ; $0.4 \mathrm{~mol} \%$

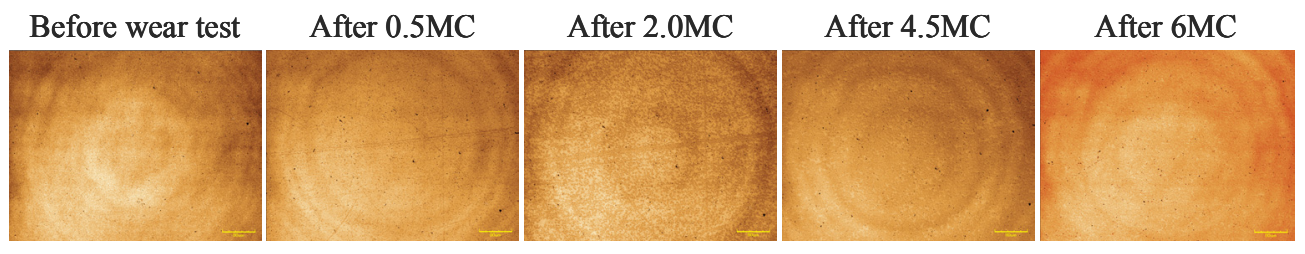

(b) Monoclinic fraction before hip simulator test ; $10 \mathrm{~mol} \%$

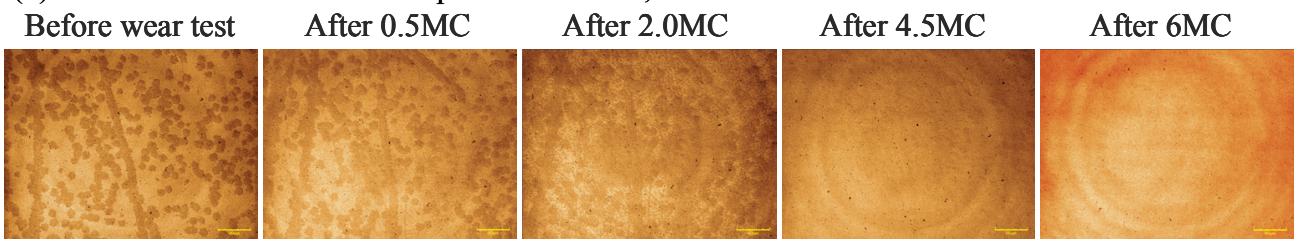

Fig. 6 Changes in surface morphology of (a) non-aged and (b) $10 \mathrm{~mol} \%$ zirconia femoral heads during hip simulator test.

In the non-aged and $10 \mathrm{~mol} \%$ samples after $6 \mathrm{MC}$, unlike in aging test, the monoclinic swells were not observed, in spite of having almost same monoclinic fraction of about 10 $\mathrm{mol} \%$. In the 2.0 and 4.5 MC, there are larger numbers of monoclinic swells with smaller in size compared with those in aging test. On the other hand, in the 50 and $80 \mathrm{~mol} \%$ zirconia femoral heads, monoclinic fraction dose not increase during the whole hip simulator test. And no significant changes in laser microscope images were observed. From these results of XRD study and laser microscope observation, it was confirmed that surface morphologies of zirconia femoral heads after aging and simulator tests were clearly different and the amounts of monoclinic phase covering the surface were not equal, even if the same monoclinic fraction was calculated by XRD patterns. Schematic images of kinetics of phase transformation during aging test and hip simulator test are shown in Fig. 7.

(a) Aging test

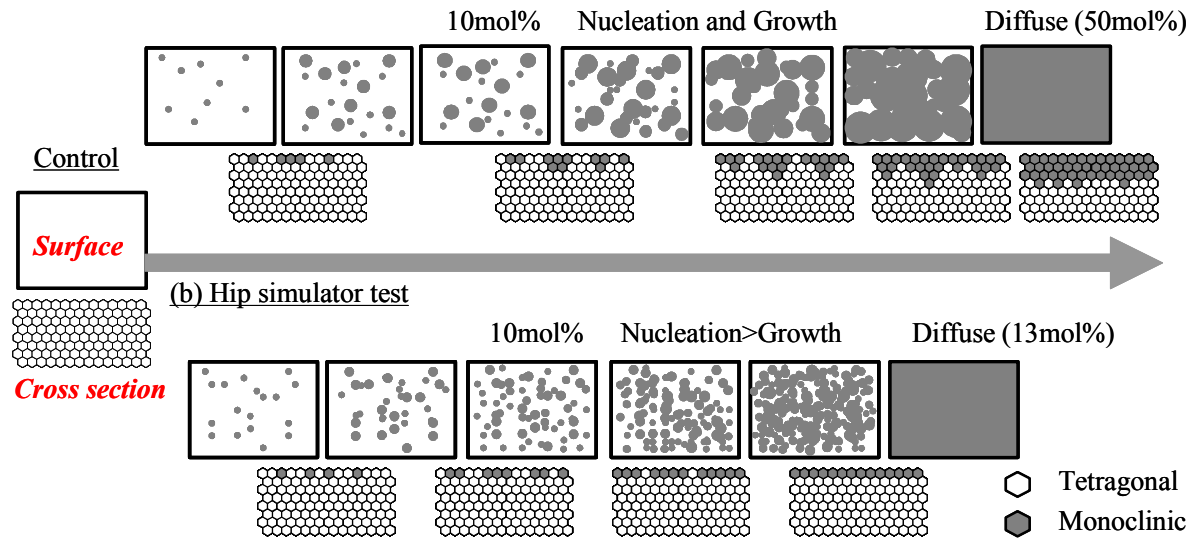

Fig. 7 Schematic image of kinetics of phase transformation of zirconia during (a) aging test and (b) hip simulator test.

(a)Kinetics of phase transformation during aging test

The monoclinic swells gradually spread out neighborhood and finally covered the surface with increasing aging time. And then monoclinic phase was grown toward inside

(b) Kinetics of phase transformation during hip simulator test

The nucleation occurred frequently and the surface is covered with monoclinic thin layer at the initial stage of phase transformation by influence of friction. 
Typical SEM images and of UHMWPE wear particles and frequency of particle distribution are shown in Fig 8 and Fig 9, respectively. From the result of UHMWPE particle collected after 0.5 MC, UHMWPE wear particle size for $10 \mathrm{~mol} \%$ aged zirconia femoral was distributed toward large particle size. However, from the result of UHMWPE wear particle collected after 5.0 MC, there were no relationship between monoclinic fraction on the zirconia femoral heads and distribution of UHMPE wear particle. It was suggested that there were relationship between surface morphology and distribution of UHMWPE wear particle, in the case of UHMWPE wear particle collected after $0.5 \mathrm{MC}$.

(a) $0.5 \mathrm{MC}$

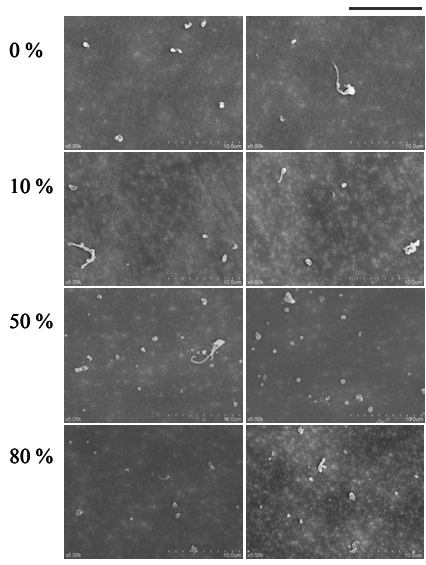

(b) $5.0 \mathrm{MC} \quad 10 \mu \mathrm{m}$

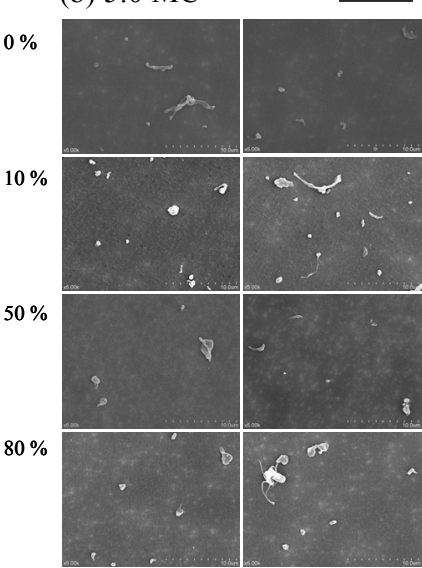

Fig. 8 SEM images of UHMWPE wear particle collected after (a) $0.5 \mathrm{MC}$ and (b) 5.0 MC.

(a) $0.5 \mathrm{MC}$

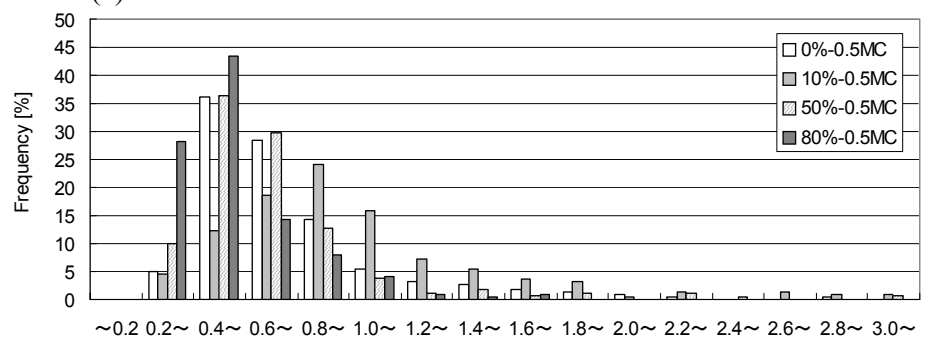

(b) $5.0 \mathrm{MC}$

Equivalent Circle Diameter $(\mu \mathrm{m})$

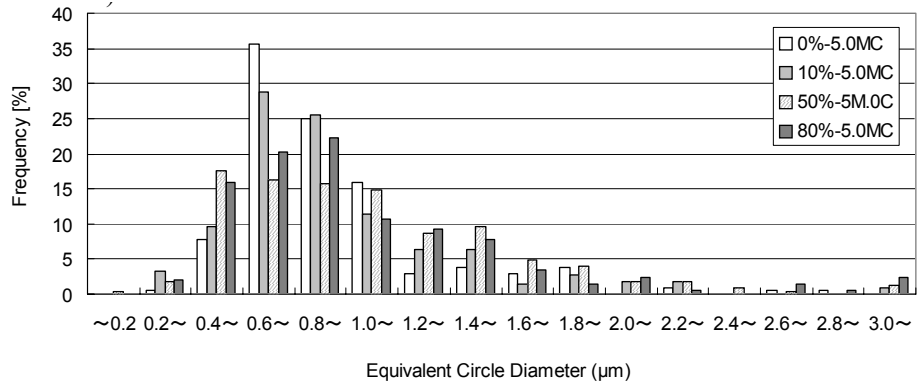

Fig. 9 Distribution of equivalent circle diameter of UHMWPE wear particle collected after (a) $0.5 \mathrm{MC}$ and (b) 5.0 MC.

Cubic ratio and tetragonality obtained by Rietveld analysis as a function of sintering temperature are shown in Fig. 10 and Fig. 11, respectively. There were about 16 to $20 \mathrm{wt} \%$ cubic phase. Tetragonality was closed to 1 and cubic ratio decreased with a decrease in sintering temperature. 


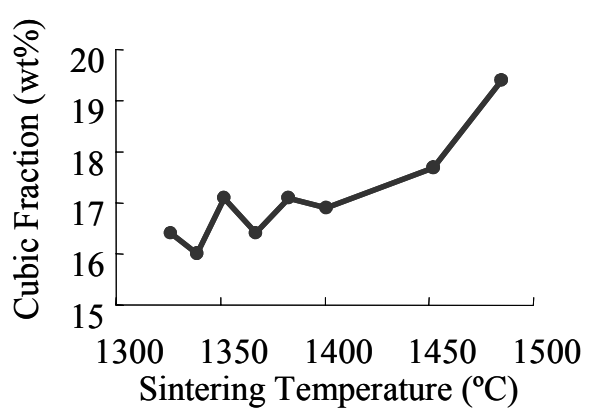

Fig. 10 Cubic ratio as a function of sintering temperature

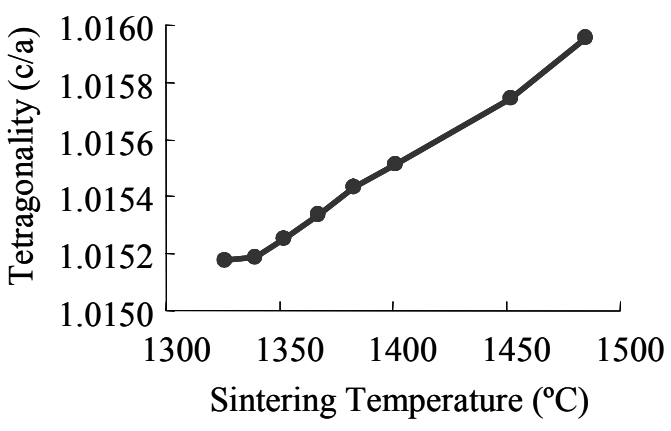

Fig. 11 Tetragonality as a function of sintering temperature

Kinetics of phase transformation during aging test experienced a Mehl-Avrami-Johnson (MAJ) equation, which properly described both nucleation and growth of monoclinic domains $^{22}$. The calculated results by MAJ equation are added, with a gray line in Fig 3.

$$
V_{\mathrm{m}}=a\left(1-\exp \left(-(b t)^{n}\right)\right)
$$

where $t$ is the aging duration time, $a$ is a constant that equals 1 when the initial monolithic fraction is zero and final monoclinic fraction is $100, b$ is a parameter that is directly proportional to the $1 / n$ power of the nucleation rate of monoclinic nuclei per unit time and area, and $n$ is an exponent which was found to range between 0.3 and 4 . In this study, saturated value was decided to $76.7 \mathrm{~mol} \%$ from the maximum value of this experimental result. The parameters $b$ and $n$ calculated from above conditions were 0.019 and 1.9 , respectively.

\section{Discussion}

In the case of aging test under hydrothermal environment, it was confirmed that a progress model of phase transformation is according to MAJ equation. Phase transformation started from some unstable tetragonal phase and spread to neighborhood region by effects of the strain arisen from volume expansion and the temperature. Moreover, a progress of phase transformation to inside from surface also occurred depending on the aging duration time. On the other hand, in the case of phase transformation during hip simulator test, larger number of monoclinic swells were observed compared to that of aging test. It is believed that the generation of monoclinic swells occurred at more activity than the spread from origin of monoclinic phase to neighborhood region in the case of phase transformation during hip simulator test. Tetragonal crystal with several lattice content presences in one sintered body. In Rietveldt analysis, the lattice content was calculated. From the result of Rietveld analysis, the lattice constant of tetragonal crystal increases with increasing sintering temperature. In the case of high temperature sintering such as zirconia without HIP treatment, the tetragonality is larger than that of zirconia with low sintering temperature. Moreover, cubic ratio of zirconia without HIP treatment increases compared to that of zirconia with HIP treatment. It was reported that causes of increase in tetragonality and cubic ratio is related to distribution of $\mathrm{Y}$ ion. Migration of $\mathrm{Y}$ ion during sintering occurred and $\mathrm{Y}$ was localized in a cubic phase and/or a part of tetragonal phase. Moreover higher sintering temperature produced higher agglomeration of $\mathrm{Y}$ ion $^{23}$. As a result, tetragonal phase with low $\mathrm{Y}$ contents were generated in the case of zirconia sintered at higher temperature. In the case of aging test, this unstable tetragonal phase with low Y contents 
became origin of spontaneous phase transformation. It is reported that the origin of phase transformation generated according to the bellow mechanism ${ }^{24}$.

i) Attacks on the zirconia surface by lone electron pair of water molecules

ii) Cut off a part of Zr-O bonds.

ii) Accumulation of strain.

iv) Formation of nucleus of monoclinic phase

In the case of phase transformation during hip simulator test, large number of monoclinic swells was observed. It was believed that cut off of $\mathrm{Zr}$-O bonds were enhanced by the sliding in lubricant containing water. However, monoclinic fractions of $50 \mathrm{~mol} \%$ and $80 \mathrm{~mol} \%$ aged zirconia femoral heads did not increased during hip simulator test. It is believed that surface of $50 \mathrm{~mol} \%$ and $80 \mathrm{~mol} \%$ zirconia femoral heads were covered with monoclinic thin layer. So it is necessary for more progress of the phase transformation to increase in temperature. Wan et al reported that temperature of surface of zirconia femoral head raised up about $90{ }^{\circ} \mathrm{C}$ during hip simulator test ${ }^{25}$. However, it was suggested from our result that temperature of zirconia femoral head is relatively low and the enhancement of phase transformation during hip simulator test was effected by factor other than increasing temperature, because there was no significant progress of the phase transformation toward inside in accordance with MAJ equation. Ikeuchi et al reported that tribochemical reaction occurred during sliding in vitro wear condition ${ }^{26}$. Alumina and silicon nitride generated $\mathrm{AlOH}$ and $\mathrm{SiO}_{2}$, respectively. However this reaction occurred at only surface. It was suggested that tribochemical reaction during sliding condition enhances chemical reaction between water molecules and zirconia surface. From these findings, difference kinetics of the two phase transformation under presence of water was observed.

In some report about retrieved sample with high transformation ratio, the transformation over $50 \mathrm{~mol} \%$, was reported. On the other hands, changes in surface morphology in vivo do not definitely correspond to that of aging test. In retrieved samples, increase in monoclinic fraction was often observed without significant change in surface roughness. This phenomenon corresponded to the phase transformation during hip simulator study.

The phase stability of current commercially available zirconia is dramatically improved compared to that of earlier generation zirconia. Recently, various alumina and zirconia composites such as zirconia toughened alumina (ZTA) and alumina toughened zirocnia (ATZ) were developed and proposed as alternatives to the monolithic alumina and zirconia. As described above, in vivo phase stability is intricately related to the stability under aging conditions. So that reason, in zirconia contained material such as ZTA and ATZ, especially material which changes crystal structure by aging test under hydrothermal environment easily, it is necessary to take a wait-and-see approach as the changes in crystal structure during in vivo use, carefully.

\section{Conclusion}

It was found in comparison study for aging test under hydrothermal environment for 3Y-TZP and hip joint simulator test for 3Y-TZP-on-UHMWPE hip prostheses that the progress model of phase transformation during the hip simulator test was different from the progress model during aging test in hydrothermal environment. It was suggested that tribochemical reaction during sliding condition enhanced the phase transformation of zirconia 


\section{References}

1. Hench LL, Wilson J. An introduction to bioceramics. World scientific. 1993;158-159.

2. Piconi C, Maccauro G. Zirconia as a ceramic biomaterial. Biomaterials 1999;20(1):1-25.

3. Urban JA, Garvin KL, Boese CK, et al. Ceramic-on-polyethylene bearing surfaces in total hip arthroplasty. Seventeen to twenty-one-year results. J Bone Joint Surg 2001;83-A:1688-1694.

4. Kawanabe K, Tanaka K, Tamura J, et al.Effect of alumina femoral head on clinical results in cemented total hip arthroplasty: old versus current alumina.

2005;10:378-384.

5. Koo KH, Ha YC, Jung WH, Kim SR, Yoo JJ, kim HJ. Isolated fracture of the ceramic head after third-generation alumina-on-alumina total hip arthroplasty. J Bone Joint Surg Am. 2008:90(2):329-36

6. Cera News. The Magazine of CeramTec AG, Medical Products Division. 2008;2.

7. Kumar P, Oka M, Ikeuchi K, Shimizu K, Yamamuro T, Okumura H, Kotoura Y. Low wear rate of UHMWPE against zirconia ceramic (Y-PSZ) in comparison to alumina ceramic and SUS 316L alloy. J Biomed Mater Res 1991;25(7):813-28.

8. C. Piconi, G. Maccauro. Zirconia as a ceramic biomaterial. Biomaterials 1999:20:1-25

9. Sato T, Shimada M. Transformation of yttria-doped tetragonal $\mathrm{ZrO} 2$ polycrystals by annealing in water. J Am Ceram Soc 1985;68:356-359.

10. Gravie RC, Hannink RHJ, Pascoe RT. Ceramic steel?. Nature 1975;258:703-704.

11. Gupta TK, Lange FF, Bechtold JH. Effect of stress induced phase transformation on the properties of polycrystalline zirconia containing tetragonal phase. J Mater Sci 1978;13:1464-1470.

12. Evans AG, Heuer AH. Transformation toughening in ceramics; martensitic transformation in crack-tip stress fields. J Am Ceram Soc 1980;63:241-248.

13. Sato T, Ohtaki S, Endo T, Shimada M. Changes in crystalline phase and microstructure on the surface of yttria-doped tetragonal zirconia polycrystals (Y-TZP) by annealing in humid conditions. In: Somiya S, Yamamoto N, Yanagida H, editors. Advances in Ceramics vol. 24: Science and technology of zirconia III, Westwerville: The American Ceramic Society,1988. p. 501-508.

14. Chevalier J, Cales B, Drouin J M. Low-temperature aging of Y-TZP ceramics. J Am Ceram Soc 1999;82:2150-2204.

15. http://www.prozyr.com

16. Clarke IC, Manaka M, Green DD, et al: Current status of zirconia used in total hip implants. J Bone Joint Surg Am2003: 85A:73-84

17. Villetmaux F, Cales B, Blaise L. Reliability of zirconia hip joint heads: Combination of wear and aging test. Key Eng Mater 2002;218-220:561-562.

18. Haraguchi K, Sugano N, Nishii T, Miki H, Oka K, Yoshikawa H. Phase transformation of a zirconia ceramic head after total hip arthroplasty. J Bone Joint Surg Br 2001;83(7):996-1000.

19. Ikeda J, Pezzotti.G, Kondo M. Phase stability and residual stress field in nano-structured 3Y-TZP. Key Eng Mat. 2006;309-311:1227-1230.

20. Garvie RC, Nicolson PS. Phase analysis in zirconia system. J Am Ceram Soc 1972;55:303-305.

21. Clarke IC, Pezzotti G, Sakakura S. Bio-lubrication phenomena affect residual stress and phase of zirconia implants. Key Eng Mater 2003;240-242:781-784.

22. Johnson W, Mehl R. Reaction kinetics in processes of nucleation and growth. Transactions of the Metallurgical Society of AIME 1939;135:416-458. 
23. Yashima M, Ishizawa N, Yoshimura M. Application of an ion-packing model based on defect clusters to zirconia solid solutions. J Am Ceram Soc 1992;75:1541-1549.

24. Lughi V, Sergo V. Low temperature degradation -aging- of zirconia: A critical review of the relevant aspects in dentistry. Dental Materials 2010:26(8): 807-820

25. Liao Y.-S., Makellop H., Lu Z, Cambell P and Benya P. The effect of frictional heating and forced cooling on the serum lubricant and wear of UHMW polyethylene cups against cobalt-chromium and zirconia balls Biomaterials 2003;24(18): 3047-3059

26. Zhou Y. S Ikeuchi K. Ohashi M. Comparison of the friction properties of four ceramic materials for joint replacements Original Research Article. Wear, 1997 210, Issues $1-2 ; 171-177$ 